\title{
Detection of the Electrical Surface Charge Induced by Treatment of the Membrane Lipid Bilayer of Human Erythrocytes
}

\author{
Akira Tamura*, Kunihiko Morita*, Tatsuzo Fujii* and Kiyohide Kojima** \\ *Department of Biochemistry, Kyoto College of Pharmacy, Yamashina-ku \\ Kyoto 607, and ${ }^{* *}$ Cancer Research Institute, Nagoya University School of \\ Medicine, Nagoya 466, Japan
}

\begin{abstract}
The lipid bilayer of the membrane of human erythrocytes from which most sialic acid residues on the outer surface had been removed was digested by phospholipase from outside the medium or treated by the incorporation of exogenous amphiphilic lipid, then it was electrophoresed. After treatments that caused the additional exposure of the dissociable polar head group of the amphiphilic lipid to the outer medium, the electrophoretic mobility of the treated cells increased as the $\mathrm{pH}$ of the suspending medium rose. This showed that there was an increase in the negative charges in the immediate vicinity of the lipid bilayer surface of the membrane. The possible effect of the dissociation of exposed head groups in the lipids on the morphology of the membrane is discussed.
\end{abstract}

We reported previously that a change in the shape of human erythrocytes is induced by in vitro treatments of the membrane lipid bilayer $(1,8)$ and that after some treatments which liberate or form anionic head groups in the lipid molecules exposed on the outer surface of the membrane, the degree of the change in shape became markedly dependent on the $\mathrm{pH}$ of the medium $(8,9)$. We assumed that the dissociation of these polar head groups in an appropriate $\mathrm{pH}$ range might increase the repulsion among the charged groups or increase the hydration around these groups. This in turn would result in the asymmetric expansion of the outer half of the lipid bilayer, relative to the inner half because the mechanism for the change in the shape of the erythrocytes can be explained by the asymmetric expansion of one half of the bilayer according to the "bilayer couple hypothesis" (7). To prove the validity of our assumption, we have demonstrated by cell electrophoresis that these treatments do cause the increased exposure of dissociable head groups on the bilayer outer surface, and that these groups become negatively charged in the $\mathrm{pH}$ range of their dissociation. We used desialydated erythrocytes which had been treated with neuraminidase to remove most of the sialic acid residues on their outer surfaces because the abundant negative charges of these residues must prevent the electrophoretic detection of the relatively weak negative charges contributed by the polar head groups of the membrane lipids.

Abbreviations used: PBS, phosphate buffered saline; HEPES, 2-[4-(2-hydroxyethyl)-1-piperazinyl] ethanesulfonic acid; MES, 2-morpholinoethanesulfonic acid; PLase, phospholipase; PC, phosphatidylcholine; TLC, thin layer chromatography. 


\section{MATERIALS AND METHODS}

Erythrocytes. Fresh human blood from the same normal donor was used in all experiments. Several milliliters of blood were drawn into a glass syringe containing heparin. The erythrocytes were washed three times with isotonic saline containing $10 \mathrm{mM}$ phosphate buffer solution (PBS), pH 7.4 then resuspended in PBS.

Treatment of erythrocytes with neuraminidase. A 0.25 unit portion of neuraminidase from Arthrobacter ureafaciens (Marukin Shoyu Co., Ltd.) per milliliter of cells was added

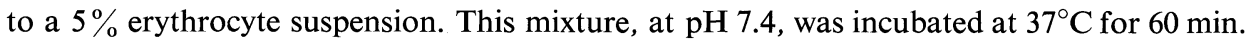
The treated cells were centrifuged, washed three times with $1 \%$ bovine serum albumin (Fr. V, Armour Pharmaceutical Co.) solution in PBS then resuspended in PBS. Sialic acid was determined according to the method of Massamiri et al. (4).

Treatment of erythrocytes with phospholipases. Ten volumes of a solution of phospholipase $\mathrm{A}_{2}$ from bee venom (Sigma Co.), phospholipase C from Clostridium perfringens (P.L. Biochemicals Inc.) or phospholipase D from Streptomyces chromofuscus (a gift from Toyo Jozo Co.) in Tris-saline containing $5 \mathrm{mM} \mathrm{CaCl}_{2}$ were added to one volume of a $50 \%$ erythrocyte

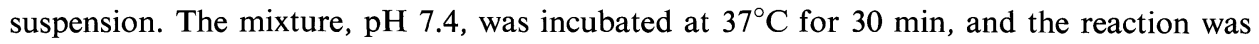
stopped by adding 11 volumes of isotonic saline containing $10 \mathrm{mM}$ EDTA. Phospholipids were determined after isolation by two-dimensional TLC, and fatty acids were determined by gas chromatography after separation on a TLC plate, as described previously $(1,8)$.

Treatment of erythrocytes with exogenous lipids. One volume of palmitic acid or dilauroylphosphatidic acid emulsion in PBS was added to one volume of $20 \%$ erythrocyte suspension in PBS. This mixture, pH 7.4, was incubated at $37^{\circ} \mathrm{C}$ for $20 \mathrm{~min}$. The amount of lipid incorporated into the cell was determined as described in the preceding section.

Cell electrophoresis. Isotonic HEPES (2-[4-(2-hydroxyethyl)-1-piperazinyl] ethanesulfonic acid) and MES (2-morpholinoethanesulfonic acid) buffer solutions were prepared by adding $\mathrm{NaCl}$ to $100 \mathrm{mM}$ solution of HEPES and MES; isotonicity was determined with an osmometer. Isotonic media for cell electrophoresis at $\mathrm{pH} 8$ and ionic strengths of $0.07,0.035$ and 0.0175 were prepared by mixing one volume of isotonic HEPES buffer, $\mathrm{pH} 8$, with 1.5 , 4 and 9 volumes of a $5 \%(\mathrm{w} / \mathrm{v})$ sorbitol solution. Isotonic media for cell electrophoresis with an ionic strength of 0.0175 at pHs 6,7 and 8 were prepared by adding one volume of isotonic MES buffer, $\mathrm{pH} 6$ or isotonic HEPES buffer, $\mathrm{pH} 7$ and 8 , to 9 volumes of $5 \%(\mathrm{w} / \mathrm{v})$ sorbitol solution. The electrophoretic mobility of individual cells was measured at $25 \pm 0.5^{\circ} \mathrm{C}$ with a Zeiss cytopherometer. Each cell was allowed to move $16 \mu \mathrm{m}$ in either direction following the reversal of current in the media. Mobility was determined from separate experiments on 15 cells for each set of conditions and was calculated in $\mu \mathrm{m} \cdot \mathrm{s}^{-1} \cdot \mathrm{V}^{-1} \cdot \mathrm{cm}$.

\section{RESULTS}

Human erythrocytes were treated with neuraminidase which removed about $96 \%$ of the sialic acid residues in their membranes. The electrophoretic mobility of these desialydated, intact erythrocytes is plotted against the ionic strength of the isotonic suspending medium at $\mathrm{pH} 8$ (Fig. 1). Mobility was very low at an ionic strength of 0.070 , but it increased considerably at 0.0175 to a level that was easily measurable by ordinary mobility determination. After further treatment with phospholipase $\mathrm{A}_{2}$, which hydrolyzed about $40 \%$ of the phosphatidylcholine molecules in the outer leaflet of the membrane lipid bilayer, the cells were electrophoresed at various ionic strengths. Fig. 1 clearly shows that a mobility twice as high as that of the original desialydated cells was obtained at ionic strengths of 0.0175 and 0.035 , but not at 0.070 . In contrast, 


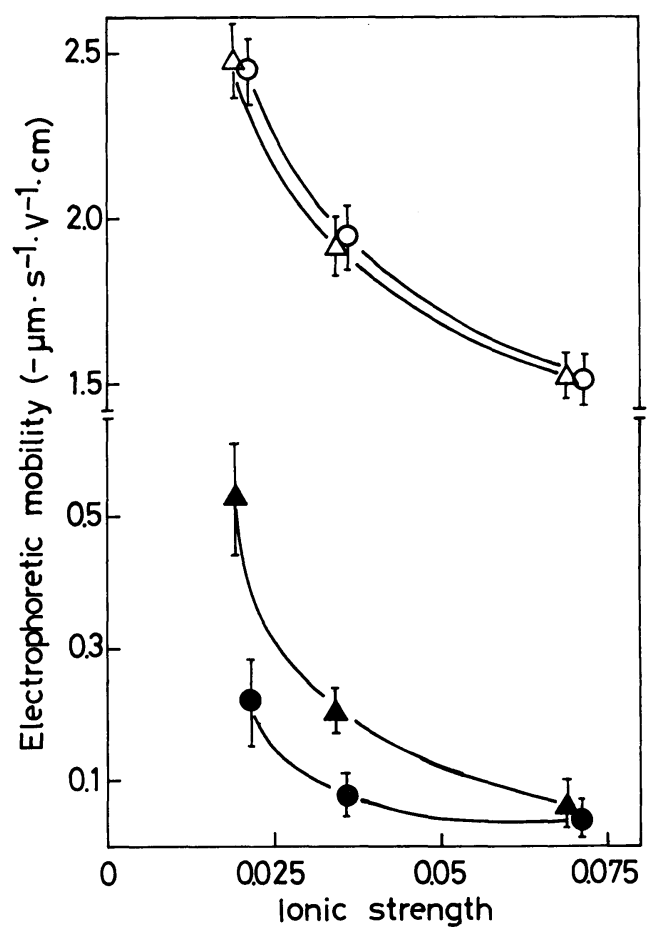

Fig. 1. Dependence of the electrophoretic mobility of desialydated and intact erythrocytes with or without phospholipase $A_{2}$ treatment on the ionic strength values of the suspending medium. Desialydated ( $(\bullet)$ and intact $(\bigcirc)$ erythrocytes, and after further phospholipase $A_{2}$ treatment $(\boldsymbol{\Delta}$ and $\triangle$ ). Electrophoresis was at $\mathrm{pH}$ 8. Each point represents the mean value for 15 cells. The length of the vertical bar is the S. D.

intact erythrocytes had very high mobility, and treatment with phospholipase $A_{2}$ did not produce any appreciable increase in their mobility. This preliminary experiment showed that an ionic strength of 0.0175 should be used in the following experiments.

Five different types of treatments of the membrane lipid bilayer were applied to the desialydated erythrocytes, after which increases in the fatty acid or phosphatidic acid contents of the treated cells were determined. Incubation with $40 \mu \mathrm{M}$ palmitic acid and with $50 \mu \mathrm{M}$ dilauroylphosphatidic acid caused the incorporation of an appreciable amount of fatty acid and of phosphatidic acid into the cells (Table I), whereas incubation with phospholipase $\mathrm{A}_{2}$ or with phospholipase D liberated considerable amounts of fatty and phosphatidic acids as a result of the hydrolysis of $44-48 \%$ of the phosphatidylcholine in the outer leaflet of the bilayer (1). Phospholipase C treatment produced neither fatty nor phosphatidic acids (it must split phosphatidylcholine into diacylglycerol and phosphorylcholine), although it hydrolyzed $30 \%$ of the phosphatidylcholine in the outer leaflet.

These treated erythrocytes and the control cells (desialydated erythrocytes) then were electrophoresed in isotonic media with an ionic strength of 0.0175 at $\mathrm{pH} 6,7$ and 8. Results are given in Fig. 2. Cells with increased fatty acid contents, as a result of palmitic acid or phospholipase $\mathrm{A}_{2}$ treatment, had markedly higher mobility than the control at an alkaline $\mathrm{pH}$, particularly in the medium at $\mathrm{pH} 8$. Similar results were 
TABLE 1. INCREASE IN THE CONTENT OF ACIDIC AMPHIPHILIC LIPID IN THE MEMBRANES OF HUMAN ERYTHROCYTES AS A RESULT OF TREATMENT OF THE MEMBRANE LIPID BILAYER

\begin{tabular}{|c|c|c|c|}
\hline \multirow{2}{*}{ Treatment } & \multicolumn{2}{|c|}{$\begin{array}{l}\text { Increase in lipid content } \\
(\mathrm{nmol} / \mathrm{ml} \text { cells })\end{array}$} & \multirow{2}{*}{$\begin{array}{l}\text { Hydrolysis } \% \\
\text { of PC in the } \\
\text { outer half of the } \\
\text { lipid bilayer }\end{array}$} \\
\hline & Fatty acid & Phosphatidic acid & \\
\hline Palmitic acid $(40 \mu \mathrm{M})$ & 254 & - & - \\
\hline Phospholipase $\mathrm{A}_{2}$ * & 417 & - & 44 \\
\hline $\begin{array}{l}\text { Dialuroylphosphatidic } \\
\text { acid }(50 \mu \mathrm{M})\end{array}$ & - & 284 & 一 \\
\hline Phospholipase D* & - & 459 & 48 \\
\hline Phospholipase $C^{*}$ & - & - & 30 \\
\hline
\end{tabular}

$* 40,200$ and 0.2 units respectively, of phospholipase $A_{2}, D$ and $C$ were used per ml of packed cells.

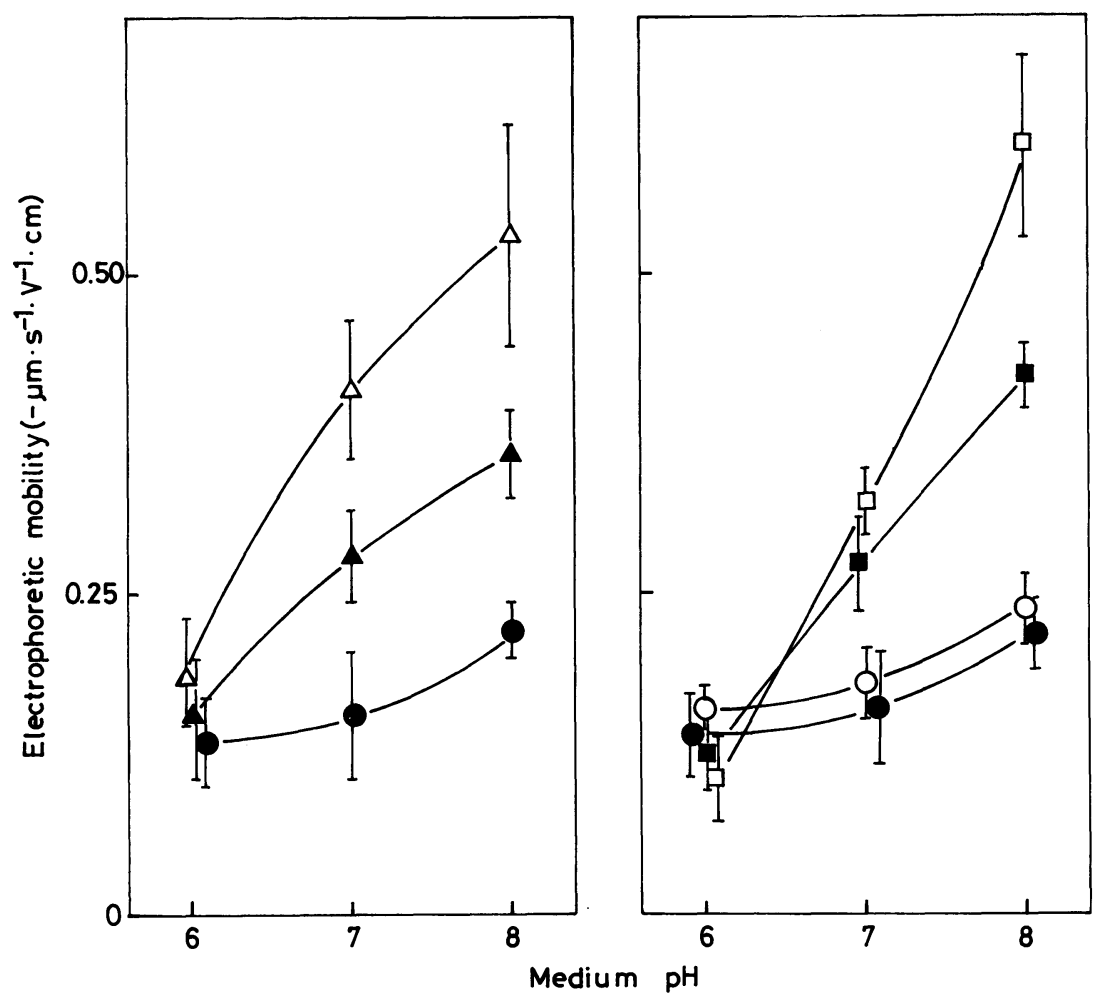

Fig. 2. Dependence of the electrophoretic mobility of desialydated erythrocytes with or without (the control) treatment of the lipid bilayer on the $\mathrm{pH}$ values of the suspending medium. Desialydated erythrocytes (the control) $(\bullet)$ and after phospholipase $A_{2}(\triangle)$, palmitic acid $(\Delta)$ and phospholipase D ( $\square$ ), dilauroylphosphatidic acid $(\square)$ and phospholipase $C$ treatment $(\bigcirc)$. Electrophoresis was at an ionic strength of 0.0175 . Each point represents the mean value for 15 cells. The length of the vertical bar is the $S$. $D$. 

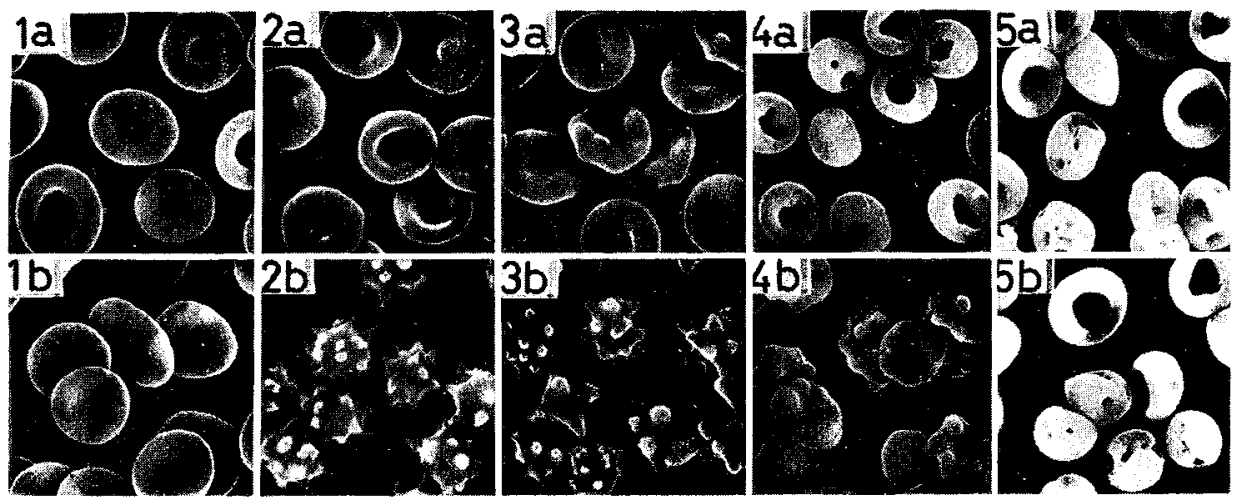

Fig. 3. Effect of the $\mathrm{pH}$ of the suspending medium on erythrocyte shape. Desialydated erythrocytes (the control) (1) were treated with $40 \mu \mathrm{M}$ palmitic acid (2), $50 \mu \mathrm{M}$ dilauroylphosphatidic acid (3), 200 units per $\mathrm{ml}$ packed cells of phospholipase $\mathrm{D}$ (4) or 0.2 units per $\mathrm{ml}$ packed cells of phospholipase $\mathrm{C}(5)$. Treated cells were suspended in a medium of $\mathrm{pH} 6$ (a) or $\mathrm{pH} 8$ (b).

obtained with desialydated cells which had an increased phosphatidic acid content after treatment with dilauroylphosphatidic acid or phospholipase D. In contrast, the mobility of the control cells and cells treated with phospholipase $\mathrm{C}$ showed no $\mathrm{pH}$ dependence, both had almost identical electrophoretic mobility at all $\mathrm{pH}$ values tested.

After desialydated erythrocytes had been treated with exogenously added PLases or amphiphilic lipids, they were placed in a medium at $\mathrm{pH} 6$ or $\mathrm{pH} 8$ (Fig. 3). The shape of the palmitic acid or dilauroylphosphatidic acid-treated cell was altered at $\mathrm{pH} 6$ from that of a weak echinocyte ( $2 \mathrm{a}$ or $3 \mathrm{a})$ to a spheroechinocyte at $\mathrm{pH} 8$ (2b or $3 b$ ). A similar shape change was observed for PLase Az-treated cells (deta not shown). But, stomatocytes observed at $\mathrm{pH} 6(4 \mathrm{a})$ in the PLase D-treated cells became echinocytes at $\mathrm{pH} 8(4 \mathrm{~b})$. In contrast, the morphology of untreated (biconcave disc, 1a and $1 \mathrm{~b}$ )and PLase C-treated cells (stomatocyte, 5a and $5 \mathrm{~b}$ ) was only mildly dependent on the $\mathrm{pH}$.

\section{DISCUSSION}

The electrophoretic mobility of human erythrocytes estimated in a commonly used medium of relatively high ionic strength reflects almost exclusively the negative charges of the sialic acid residues in the peripheries of the polypeptide chains protruding from the outer surface of the membrane. An increase in the electrophoretic mobility of the cell as the ionic strength of the measuring buffer solution decreases has been observed in erythrocytes and tumor cells. The internal ionized groups on the cell surface have a greater effect at low ionic strengths $(2,3)$. With decreasing ionic strength, the effective thickness of the double ionic layer at $25^{\circ} \mathrm{C}$ is $3.06 \times$ (ionic strength) ${ }^{-1 / 2} \dot{A}$, as calculated from Debye-Hückels equation (5). In our present electrophoretic study, we wanted to compare the electric charges of the dissociable polar head groups of the membrane lipids exposed to external medium at various $\mathrm{pH}$ values, thus we used human erythrocytes deprived of most (ca. 96\%) of their sialic acid residues and an isotonic medium with a low ionic strength, 0.0175 . Under these conditions, when we used the thickness of the ion atmosphere as an approximate estimation of the electrokinetic plane of shear, theoretically we could detect the 
ionized groups in the peripheral 0-23 $\AA$ zone of shear. The dependency of electrophoretic mobility on ionic strength suggests that the polar head groups of the membrane lipid may exist in a zone deeper than $20 \AA$ in desialydated erythrocytes.

The desialydated erythrocytes were treated as follows: incubation with palmitic acid or a medium-chain phosphatidic acid which is easily incorporated into the membrane (8); incubation with added phospholipase $\mathrm{A}_{2}$ or D which hydrolyzes phospholipid molecules (mainly PC) in the outer leaflet of the lipid bilayer and liberates the fatty acid carboxyl group or the second $\mathrm{OH}$ group of phosphoric acid $(1,9)$; and incubation with phospholipase $\mathrm{C}$ which removes the phosphorylcholine moiety from the phospholipids in the outer leaflet. The first two treatments were to increase the number of anionic groups of the amphiphilic lipids exposed to the external medium, whereas the last treatment served as a control in the sense that it produced no dissociable polar head group in situ when the membrane lipid was hydolyzed.

A definite increase in electrophoretic mobility was observed on the specimen after treatment 1 or 2 in a medium of $\mathrm{pH} 7$ or 8 , but not in medium of $\mathrm{pH} 6$. In contrast, at all the pHs tested, after treatment 3 the erythrocytes showed mobility that was almost identical with that of the control cells which had had no treatment. These facts indicate that the increased mobility at these alkaline $\mathrm{pH}$ values reflects an increase in the negative charges produced by dissociation of the anionic head groups of the amphiphilic lipid molecules added or formed in the outer half of the lipid bilayer in the $\mathrm{pH}$ range of 6 to 8 because the $\mathrm{COOH}$ group of a long-chain fatty acid and the second $\mathrm{OH}$ of the phosphoric acid in the phosphatidic acid molecules in a monolayer or bilayer state dissociated in this $\mathrm{pH}$ range (6).

We demonstrated that the shape of desialydated erythrocyte becomes markedly dependent on the $\mathrm{pH}$ of the suspending medium after treatment of the cell membrane lipid bilayer to expose additional anionic groups of lipids. This confirms similar results obtained for native erythrocytes with intact sialic acid residues $(8,9)$. The treated cells became crenated to a greater extent at $\mathrm{pH} 8$ than at $\mathrm{pH} 6$ when treated with PLase $\mathrm{A}_{2}$, palmitic acid or dilauroylphosphatidic acid. But, cells which were invaginated at $\mathrm{pH} 6$ by PLase D treatment became crenated at $\mathrm{pH} 8$. In contrast, the normal biconcave disc shape of the controls and the invaginated shape of the PLase $\mathrm{C}$-treated cells remained about the same at $\mathrm{pH} 6$ and 8. These results support our previous assumption $(8,9)$ that the negative charges of dissociable head groups of membrane lipids exposed to the external medium effect the membrane conformation and shape to a considerable extent, probably due to expansion of the outer half of the bilayer by increased repulsion among the charged groups or by increased hydration around these groups. Interestingly, removal of sialic acid residues, which contribute the majority of negative charges on the outer surface of the membrane, has no effect on the shape of the erythrocytes (9). Electrical charges in the immediate vicinity of the membrane bilayer surface may be what is important for the regulation of the membrane conformation and, hence, cell shape.

Acknowledgements. This work was supported in part by a Grant-in-Aid (No. 237051) for Scientific Research and by Grants-in-Aid for Cancer Research from the Ministry of Education, Science and Culture of Japan.

\section{REFERENCES}

1. Fuji, T. and A. TAmura. Asymmetric manipulation of the membrane lipid bilayer of intact 
human erythrocytes with phospholipase A, C, or D induces a change in cell shape. J. Biochem. (Tokyo) 86, 1345-1352, 1979

2. Herd, D.H. and G.V.F. Seaman. The influence of $\mathrm{pH}$ and ionic strength on the electrokinetic stability of human erythrocyte membrane. J. Gen. Physiol. 43, 635-654, 1960

3. Maddy, A.H. The chemical organization of the plasma membrane of animal cells. Int. Rev. Cytol. 20, 1-65, 1966

4. Massamiri, Y., G. Durand, A. Richard, J. Feger and J. Agneray. Determination of erythrocyte surface sialic acid residues by a new colorimetric method. Anal. Biochem. 97, 346-351, 1979

5. Sato, C., K. Kojima and K. Nishizawa. Translocation of hyaluronic acid in cell surface of cultured mammalian cells after X-irradiation and its recovery by added adenosine triphosphate. Biochim. Biophys. Acta 470, 446-452, 1977

6. SeimiYA, T. and S. OHKI. Ionic structure of phospholipid membranes, and binding of calcium ions. Biochim. Biophys. Acta 298, 546-561, 1973

7. Sheetz, M.P. and S.J. Singer. Biological membranes as bilayer couples. A molecular mechanism of drug-erythrocyte interactions. Proc. Natl. Acad. Sci. U.S.A. 71, 4457-4461, 1974

8. Tamura, A., K. Morita and T. FujII. Interaction of added amphiphilic lipids with the membrane of intact human erythrocytes to induce change in the cell shape. J. Biochem. (Tokyo), 91, 73-78, 1982

9. TAmURA, A. and T. FuJII. Roles of charged groups on the surface of membrane lipid bilayer of human erythrocytes in induction of the shape change. J. Biochem. (Tokyo), 90, 629-634, 1981

(Received for publication, September 18, 1981) 